\title{
Die operative Behandlung von Gelenkknorpeldefekten unter besonderer Berücksichtigung der autologen Knorpelzelltransplantation: Grundlagen - Ergebnisse - Ausblick
}

\author{
K. Weise, T. Krackhardt, Ch. Gaissmaier
}

\section{Zusammenfassung}

Die Behandlung von Defekten des Gelenkknorpels ist problematisch, da er nur ein geringes Regenerationspotential besitzt. In Abhängigkeit des Defektausmaßes und der -ursache werden derzeit unterschiedliche Verfahren zur operativen Behandlung von Knorpelschäden eingesetzt. Die in der Vergangenheit etablierten Behandlungsmethoden können zwar eine Defektauffüllung bewirken, meist handelt es sich jedoch um ein narbiges Bindegewebe minderwertiger Qualität, das häufig einem vorzeitigen Verschleiß unterliegt. Diese unbefriedigenden Ergebnisse haben daher zu großen Anstrengungen geführt, neue Therapieverfahren mit kurativer Zielsetzung zu entwickeln. Die Vermehrung körpereigener Knorpelzellen unter Laborbedingungen mit anschließender Retransplantation der angezüchteten Zellen stellt einen solchen Therapieansatz dar. Die eindrucksvollen Ergebnisse dieses auch als autologe Knorpelzelltransplantation (ACT) bezeichneten Verfahrens, die bisher vor allem von einer schwedischen Arbeitsgruppe berichtet wurden, konn- ten von anderen Autoren nicht immer bestätigt werden. Unsere ersten eigenen Erfahrungen mit der ACT deuten darauf hin, dass bei adäquater Transplantatqualität und Operationstechnik ein gutes Ergebnis der ACT vor allem durch die korrekte lndikationsstellung bestimmt wird. An der Berufsgenossenschaftlichen Unfallklinik Tübingen wird die ACT daher vor allem bei jungen Patienten mit großen Knorpeldefekten traumatischer Ursache eingesetzt, bei denen etablierte Verfahren, wie z.B. die Pridiebohrung, erfahrungsgemäß überfordert sind. Da das Ergebnis der autologen Knorpelzelltransplantation jedoch von einer Vielzahl unterschiedlicher und komplexer Faktoren abhängig ist, kann ihre Anwendung nur von hierfür ausgebildeten Ärzten und an spezialisierten Zentren unter Gewährleistung definierter Qualitätskriterien empfohlen werden. Eine Erweiterung des begrenzten Indikationsspektrums der ACT wird aber nur durch eine Weiterentwicklung des Verfahrens im Sinne der artifiziellen Herstellung eines biohybriden Knorpel- oder Knorpel-Knochenimplantats erreicht.

\section{Einleitung}

Der hyaline Gelenkknorpel kann nach traumatischer Schädigung bzw. infolge einer anlagebedingten Ernährungsstörung oder bei ausgeprägtem Verschleiß aufgrund arthrotischer Veränderungen bis heute noch nicht vollwertig ersetzt werden. Derartige Veränderungen des Gelenkknorpels stellen im klinischen All-

OP-JOURNAL 2000; 16: 150-159

(c) Georg Thieme Verlag Stuttgart · New York tag ein noch weitgehend ungelöstes Problem dar. Infolge der hohen lnzidenz von Knorpelschädigungen und der daraus resultierenden Behandlungsbedürftigkeit einer Vielzahl von Patienten, sowie angesichts der bisher etablierten, den Ansprüchen an eine völlige Ausheilung kaum gerecht werdenden Therapieverfahren, sind große Anstrengungen unternommen worden, neue Techniken zum Knorpelersatz zu entwickeln. Die Vermehrung körpereigener Knorpelzellen unter Laborbedingungen mit anschließender Retransplantation der angezüchteten Zellen stellt einen solchen Therapieansatz dar.
Im Idealfall soll es mit dieser Behandlungsmethode möglich sein, bei tiefen Knorpelschäden vollwertiges Ersatzgewebe im Defektlager $\mathrm{zu}$ regenerieren und sich nicht mit einer Art „faserigem Ersatzknorpel“ bzw. einer Defektheilung zufrieden zu geben. Die Vorstellung dabei ist, dass Knorpelkrater mit einer Suspension aus körpereigenen Knorpelzellen aufgefüllt werden, die ihrerseits in der Lage sind, die komplexe Struktur des hyalinen Gelenkknorpels nachzubilden. Dass ein solcher Anspruch an die Chondrozytentransplantation klinisch, labortechnisch und operativ nur schwer umzusetzen ist, kann leicht nachvollzogen werden.

Seit 1994 haben verschiedene Autoren über ihre ersten klinischen Erfahrungen in der Behandlung tiefer Knorpeldefekte am Kniegelenk mittels autologer Chondrozytentransplantation (ACT) berichtet $[1,2,3]$. In der Folge hat dieses Verfahren eine zunehmende Bedeutung erlangt, nicht zuletzt durch das Engagement professioneller Anbieter aus der Industrie, die in der Lage sind, die Knorpelzellvermehrung bei einem allerdings nicht unbeträchtlichen finanziellen Aufwand in speziell ausgerüsteten Labors vorzunehmen und die erforderliche Logistik für die Verteilung bereitzustellen. Aus einzelnen Veröffentlichungen und aus Vorträgen bei wissenschaftlichen Veranstaltungen ist bekannt, dass in einer Reihe von klinischen Zentren sozusagen für den „Hausgebrauch“ Chondrozytenvermehrungen $\mathrm{zu}$ Transplantationszwecken vorgenommen werden.

Zur Zeit kann über die Wertigkeit des Verfahrens der ACT naturgemäß noch kein abschließendes, wissenschaftlich fundiertes und statistisch abgesichertes Statement abgegeben werden, da prospektive Untersuchungen über einen ausreichend langen Zeitraum bisher nicht vorgelegt werden konnten. 
Dennoch häufen sich auch mittelfristige Kontrollen, in denen die autologe Chondrozytentransplantation als ein geeignetes, weil auch über diese Zeiträume funktionierendes Verfahren, beschrieben wird. Die klinischen Erfahrungen sowie arthroskopische Kontrollen und diverse tierexperimentelle Untersuchungen scheinen dies zu bestätigen, so dass der weitere Einsatz der ACT unter kontrollierten und vor allem optimierten Bedingungen empfohlen wird.

Eigene Erfahrungen gehen bei allerdings eher kurzfristiger Beobachtungszeit in die gleiche Richtung, so dass uns das Verfahren der autologen Knorpelzellzüchtung und -transplantation bei geeigneter Indikation, optimierter Transplantatqualität und operativer Technik als gangbarer und erfolgversprechender Weg erscheint. Nach einer mehrjährigen Vorbereitungsphase mit parallel dazu ablaufenden experimentellen Untersuchungen wurde es möglich, dass in der Berufsgenossenschaftlichen Unfallklinik Tübingen in $\mathrm{Zu}-$ sammenarbeit mit dem Naturwissenschaftlichen und Medizinischen Institut der Universität die Voraussetzungen geschaffen wurden, Suspensionen aus autologen Knorpelzellen herzustellen und zu retransplantieren. Augenblicklich sind wir dabei, die gesetzlichen Vorgaben dafür zu erfüllen, dass das Verfahren auch für Anwender aus anderen klinischen Einrichtungen in Anspruch genommen werden kann.

\section{Therapiemöglichkeiten bei Knorpelschäden}

Bei traumatisch entstandenen Knorpelschäden oder bei der Osteochondrosis dissecans (OD) existiert bis heute eine Reihe von Behandlungsverfahren, die mehr oder weniger Notlösungen darstellen und in der Regel zu Defektheilungen führen. Ausnahme hiervon ist die Refixation eines größeren traumatisch entstandenen und durch Osteochondrose abgelösten Knorpel-Knochen-Fragmentes. Ein derartiger, vor allem das Kniegelenk betreffender Schaden kann durch Einpassen des Fragmentes in sein „Mausbett“ mit Refixation durch Fibrinkleber und zusätzlicher Anheftung durch resorbierbare Stifte behandelt werden. In solchen Fällen besteht eine gute Chance dauerhafter und vollständiger Heilung.

Ist jedoch eine Refixation des KnorpelKnochen-Fragmentes nicht möglich bzw. liegt ein tiefer Knorpeldefekt in der Belastungszone des Gelenkes vor, muss eines der übrigen, im weiteren näher beschriebenen Therapieverfahren gewählt werden.

\section{Anregung der Ersatzfaserknorpelbildung}

Diese auch als Pridie-Bohrung bezeichnete Technik hat zum Ziel, durch Anbohren bzw. durch Aufbrechen der subchondralen Schicht in einem Knorpeldefekt mittels eines kleinen Meißels das Einsprossen von Bindegewebszellen anzuregen, die ihrerseits im Defektbereich eine Art Ersatz(Faser-)knorpel ausbilden. Dieses Narbengewebe kann die mechanischen Eigenschaften des normalen $\mathrm{Ge}$ lenkknorpels in keiner Weise erfüllen und stellt somit nur eine Art Notlösung dar. Im weiteren Verlauf muss in derartigen Fällen mit einem Fortgang der Verschleißerscheinungen an dieser Stelle und mit der Entwicklung einer Arthrose gerechnet werden [4].

\section{Defektdeckung mit Perichondrium oder Periostlappen}

Die Technik der Übernähung eines tiefen Knorpeldefektes mittels Perichondrium oder Knochenhautlappen, z.B. entnommen vom Rippenknorpel oder der medialen Schienbeinfläche hat zum Ziel, Regenerate aus einer Mischung von Faser- und hyalinem Knorpel zu erzeugen und auf diese Weise den Knorpeldefekt auszufüllen. Ein Problem dieses Verfahrens ist, dass es bei Verwendung von Periost teilweise zu Verknöcherungen dieses Regenerates und zu dessen Ablösung im Sinne eines freien Gelenkkörpers kommen kann, so dass kein dauerhafter Therapieerfolg zu verbuchen ist.

\section{Allogene KnorpeI-Knochen- Transplantation}

In der Berufsgenossenschaftlichen Unfallklinik Tübingen bestehen bei einigen we- nigen Fällen Erfahrungen mit der Transplantation von Knorpel-Knochen-Zylindern, welche man aus Kniegelenken von Spendern gewonnen hat. Mit einem eigens dafür entwickelten Instrumentarium kann unter Verwendung einer Hohlfräse ein Knorpel-Knochen-Zylinder definierten Durchmessers entnommen und im Press-Fit-Verfahren in das durch analoges Fräsen vorbereitete Lager im Defektbereich eingebolzt werden. Der Fremdknorpel ist hierbei stufenfrei ins Niveau des umgebenden gesunden Knorpelareals einzupassen.

Mittelfristige Nachuntersuchungen weisen zufriedenstellende Ergebnisse auf, wobei die Knorpelfläche im transplantierten Areal makroskopisch zumindest keine Abstoßung oder Auflösung zeigte. Dieses Verfahren ist belastet durch einen hohen logistischen Aufwand auf Spenderseite und die bekannten Schwierigkeiten, die mit der Übertragung allogenen Gewebes verbunden sind. Zudem kann über Langzeitergebnisse bzw. die Dauerhaftigkeit der Einheilung keine zuverlässige Aussage gemacht werden.

\section{Autologe KnorpeI-Knochen- Transplantation}

Um die genannten Schwierigkeiten bei der Übertragung allogenen Materials zu vermeiden, wurde die Technik der autologen Knorpel-Knochen-Transplantation entwickelt. Mit einem speziellen lnstrumentarium können aus weniger belasteten Arealen der Femurkondylen KnorpelKnochen-Zylinder bis zu $1 \mathrm{~mm}$ Durchmesser bei einer Länge von etwa $2 \mathrm{~cm}$ entnommen und in vorbereitete Lager im Defektbereich eingebracht werden [5]. Bei dieser als Mosaikplastik bezeichneten Methode kommt es als Nebeneffekt infolge Eröffnung der subchondralen Knochenschicht zu der bereits genannten Faserknorpelbildung, so dass die neue

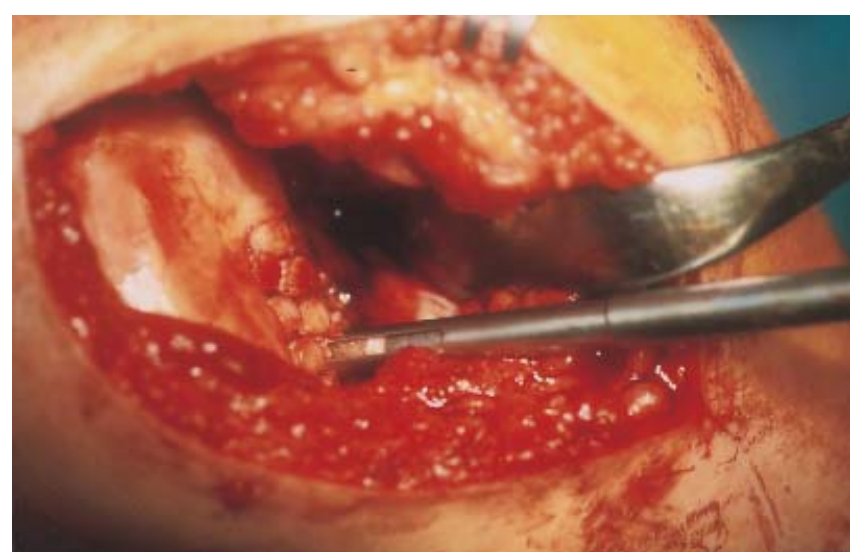

Abb.1 Bei der Mosaikplastik werden im Press-Fit-Verfahren patienteneigene Knorpel-Knochenstanzzylinder in das vorbereitete Empfängerlager des Defektbereichs eingesetzt. 


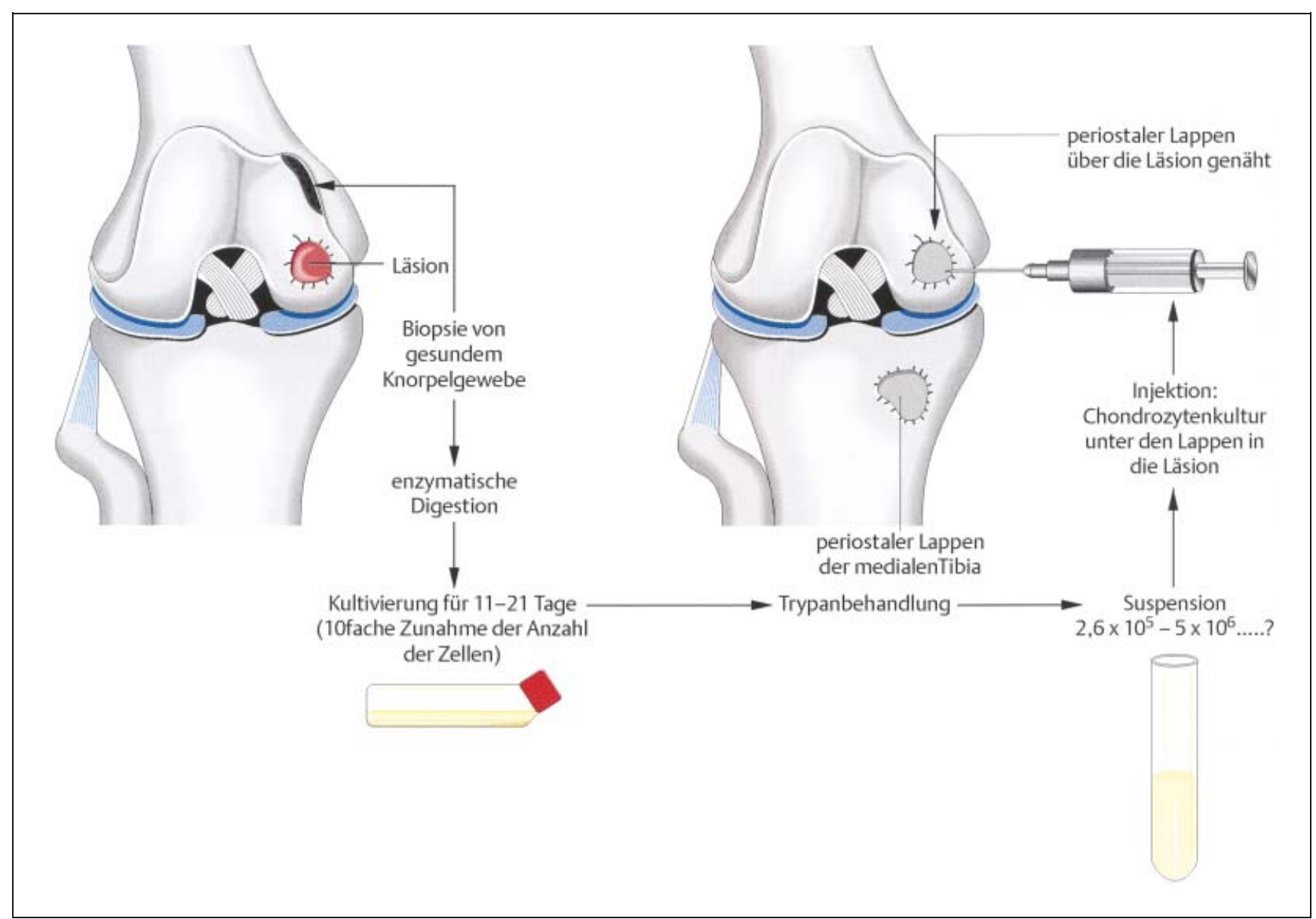

Abb. 2 Die autologe Knorpelzelltransplantation verläuft im Wesentlichen in drei Schritten. 1. Knorpelentnahme, 2. Zellanzüchtung, 3. Zellretransplantation.

Oberfläche wiederum eine Mischform beider Knorpelarten aufweist. Dennoch handelt es sich bei diesem Verfahren um eine etablierte und zumindest mittelfristig erfolgreiche Methode, die unter den bisher genannten Techniken trotz des Schadens im Entnahmebereich absoluten Vorzug genießt (Abb.1).

Therapieverfahren bei Knorpelschäden:

- Anbohrung oder Aufbrechen der subchondralen Schicht mit Ersatzfaserknorpelbildung

- Defektdeckung mit Perichondrium oder Periostlappen

- allogene Knorpel-Knochen-Transplantation

- autologe Knorpel-Knochen-Transplantation (Mosaik-Plastik)

\section{Autologe Knorpelzelltransplantation (Chondrozytentransplantation - ACT)}

Dieses Verfahren besteht aus 3 Einzelschritten, die in einem zeitlichen Intervall von 2-3 Wochen ablaufen (Abb.2). Das Grundprinzip dieser Behandlungsmethode besteht darin, dass aus einem kleinen Stückchen körpereigenen Knorpels durch spezielle labortechnische Verfahren innerhalb eines vergleichsweise kurzen Zeitraumes eine erhebliche Vermehrung der Chondrozyten bewirkt werden kann, wobei diese in Form einer Suspension in den Defekt eingebracht und unter dem Schutz eines Periostlappens zur Bildung von Knorpelgewebe veranlasst werden können. Die in der Suspension enthaltenen Knorpelzellen sind bei guter Vitalität und erhaltenem funktionellen Zustand in der Lage, mit der Produktion von Knorpelgewebe den Defekt schrittweise aufzufüllen. Die Streitfrage unter Experten ist, welche Qualität dieser Knorpel besitzt, wobei sowohl von Ersatzfaserknorpel als auch von hyalinartigem Gelenkknorpel die Rede ist.

\section{Zell- und molekularbiologische Grundlagen}

Die spezifischen Stoffwechselleistungen einer Zelle hängen eng von ihrem Differenzierungsgrad ab. Dies gilt für die meisten Zellsysteme des Bindegewebes [6,7] und in besonderer Weise für chondrogene Zellen, also für Knorpelzellen. Durch die Kultivierung der Knorpelzellen in der Monolayerkultur, einem zweidimensionalen Zellkultursystem, wie es momentan für die Anzucht von Chondrozyten zu Transplantationszwecken verwendet wird, verlieren die Knorpelzellen in Abhängigkeit der Kultivierungszeit und Passagenzahl (Passage $=$ Zellernte und erneute Aussaat in Zellkulturflaschen) ihre knorpeltypischen Eigenschaften.

Dieser Prozess, der auch als Dedifferenzierung bezeichnet wird, geht mit einem Wandel der Zellmorphologie, d. h. der äußeren Erscheinungsform, und den sekretorischen Leistungen der Zellen einher. Frisch isolierte Knorpelzellen mit einer anfänglich runden bis pflastersteinartigen Zellmorphologie verändern in der Monolayerkultur ihre Gestalt zu länglichen bis spindelförmigen ZelIformen, wie man sie z.B. von Fibroblasten kennt (Abb. 3b u.c). Mit zunehmender Dedifferenzierung nimmt die Synthese knorpeltypischer Matrixkomponenten (Kollagen 

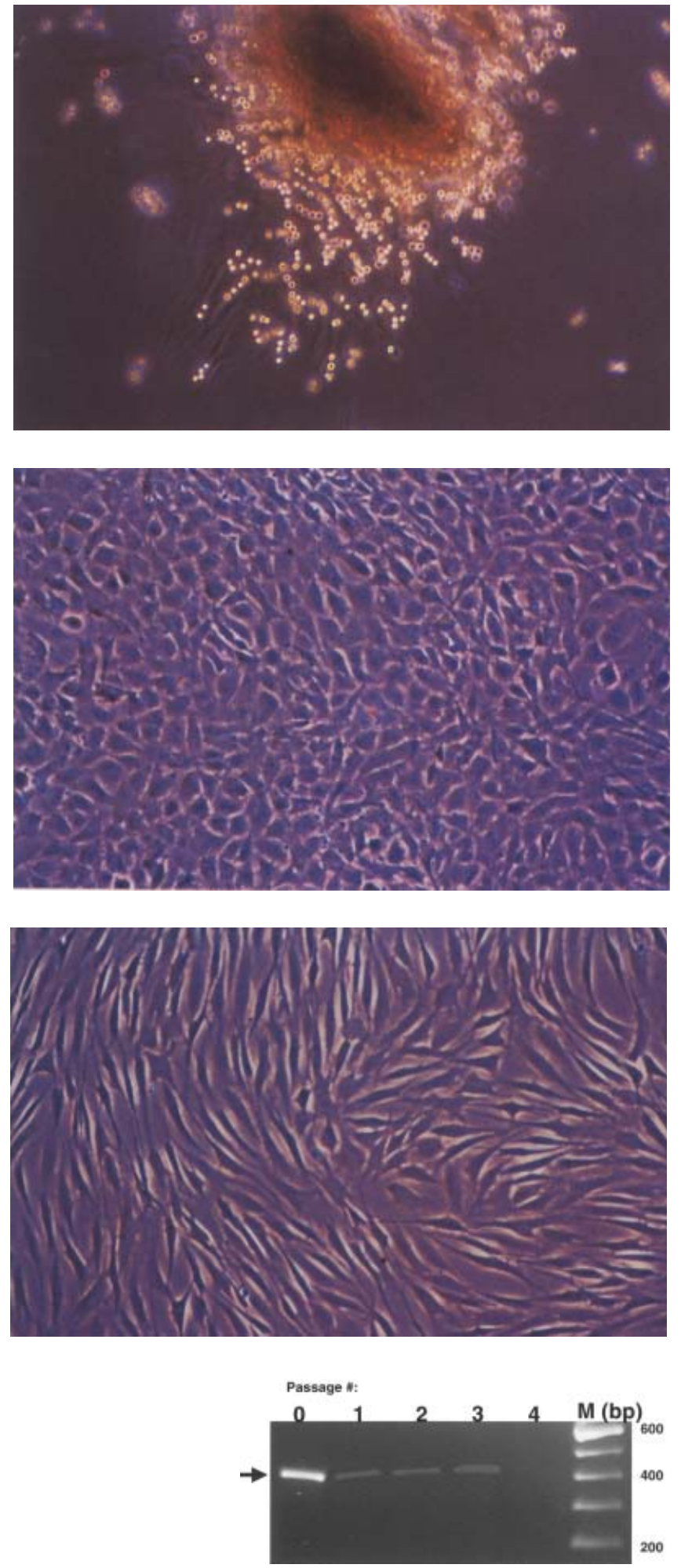

Abb. 3d Kollagen-Typ-II-RT/PCR-Analyse: Während Knorpelzellen der Primärkultur (=Passage 0 ) für KollagenTyp-II ein deutliches Transkriptionssignal aufweisen, lässt sich bei dedifferenzierten Knorpelzellen der folgenden Passagen (1 bis 4) nahezu kein Signal für die Kollagen-Typ-II-Synthese mehr erkennen.
Abb. 3a Durch fraktionierte Verdauung mit unterschiedlichen Enzymen wird die Knorpelgrundsubstanz schrittweise aufgelöst. Im Vergleich zur nicht-fraktionierten enzymatischen Zellisolation werden die Chondrozyten hierdurch weniger in ihrem primären Funktionszustand beeinträchtigt.

Abb. 3b Differenzierte Knorpelzellen weisen in der Monolayerkultur eine pflastersteinartige Erscheinungsgestalt auf.

Abb. 3c Dedifferenzierte Knorpelzellen besitzen ein länglich bis spindelförmiges Aussehen, wie man es von Fibroblasten kennt.
Typ II, IX, XI, Proteoglykane) ab (Abb. 3d und die knorpeluntypischer Kollagene (Typ I und III) zu. Überführt man in Monolayerkultur kultivierte Knorpelzellen in ein geeignetes dreidimensionales Kultursystem wie Agarose oder Alginat, können die Knorpelzellen redifferenzieren (Abb.4) [8]. Dies bedeutet, dass die Zellen ihre ehemals knorpeltypischen Eigenschaften wieder ausbilden.

Eigene Untersuchungen zur Redifferenzierungsfähigkeit humaner Knorpelzellen im Alginatsystem haben gezeigt, dass bei manchen Patienten ab Passage zwei nicht mehr alle Zellen einer Zellpopulation zu einer vollständigen chondrogenen Redifferenzierung befähigt sind oder zumindest bis zum Eintritt vollständiger Redifferenzierung auch Matrixproteine synthetisiert werden, die normalerweise im gesunden Gelenkknorpel nicht vorkommen. Die in der Literatur unterschiedlich berichteten Ergebnisse nach autologer Knorpelzelltransplantation könnten unter anderem durch diese Veränderungen der Redifferenzierungsfähigkeit in Abhängigkeit der vorausgegangenen Kultivierungsbedingungen begründet sein [9]. Subkultivierungen, die die Dedifferenzierungsprogression zusätzlich zum Faktor Zeit durch enzymatische Veränderung von zellmembranständigen Rezeptoren stimulieren, sollten daher von Knorpelzellen zu Transplantationszwecken nicht vorgenommen werden. Durch die Optimierung der Zellkulturbedingungen können Zellen mit gutem Differenzierungsniveau und spezifischer Matrixsyntheseleistung angezüchtet und anschließend transplantiert werden (Abb.5a u.b).

Eine weitere Schwierigkeit bei der Knorpelzellzüchtung und -transplantation ist, dass bei höherem Patientenalter eine abnehmende Teilungsfähigkeit bzw. mitogene Stimulierbarkeit von Zellen des Bindegewebes beobachtet wird, wodurch eine qualitativ hochwertige Zellzüchtung bei Patienten über dem 50. Lebensjahr erschwert werden kann. Neuere Studien weisen ferner darauf hin, dass es bei älteren Patienten und bei solchen mit degenerativen Veränderungen des Knorpels zu Situationen kommt, in denen die Neubildung von Knorpelgewebe verhindert wird.

Aufgrund der bisher berichteten klinischen Erfahrungen und der wissenschaftlichen Erkenntnisse, sollte die Indikation zur ACT daher unverändert auf räumlich begrenzte, überwiegend traumatisch 


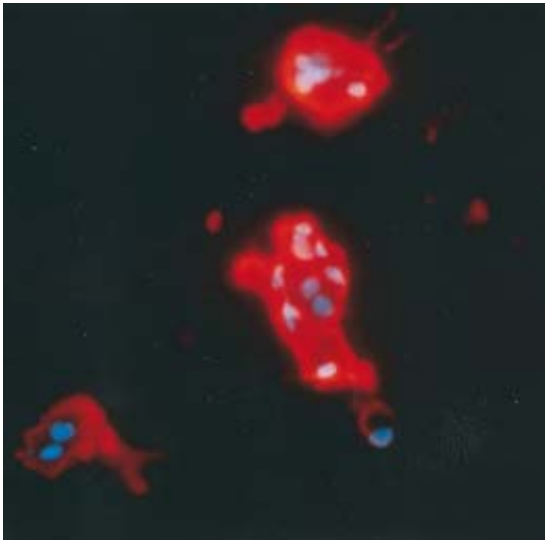

Abb.4 Dedifferenzierte Knorpelzellen können in einem geeigneten dreidimensionalen Zellkultursystem (Alginat) ihre knorpeltypischen Eigenschaften wieder ausbilden. Immunfluoreszenz-Färbung gegen Proteoglykan.

entstandene Knorpeldefekte beim jüngeren Patienten beschränkt bleiben.

\section{Technik der Knorpelzellzüichtung und -transplantation}

Das dreizeitige Verfahren der Knorpelzellentnahme, Chondrozytenzüchtung und der Retransplantation einer Knorpelzellsuspension läuft derzeit wie folgt ab [10]:

\section{Erster Schritt}

Zuerst führt man die arthroskopische Entnahme eines ca. halb kleinfingernagelgroßen Knorpelstückchens außerhalb der Belastungszone mit gleichzeitiger Blutentnahme zur Gewinnung von Patientenserum durch. Danach erfolgt die Aufbewahrung des Knorpelstückchens in einem sterilen Gefäß mit Medium. Dieses wird in ein Zellkulturlabor transportiert, das mit entsprechenden Sicherheitsstandards ausgestattet ist.

\section{Zweiter Schritt}

Nach Ankunft des Knorpels im Zellkulturlabor wird im Direktansatz und in der Anreicherung geprüft, ob eine mikrobielle Kontamination während der Knorpelentnahme oder des Transports in das Zellkulturlabor eingetreten ist. Bei positivem Keimnachweis in der durchgeführten Eingangskontrolle können keine Knorpelzellen für den hierfür betroffenen Patienten angezüchtet werden. Für die im weiteren vorzunehmende Anzüchtung und Vermehrung der Knorpelzellen müssen diese unter sterilen Bedingungen aus dem Knorpelstückchen, d.h. der Knorpel-

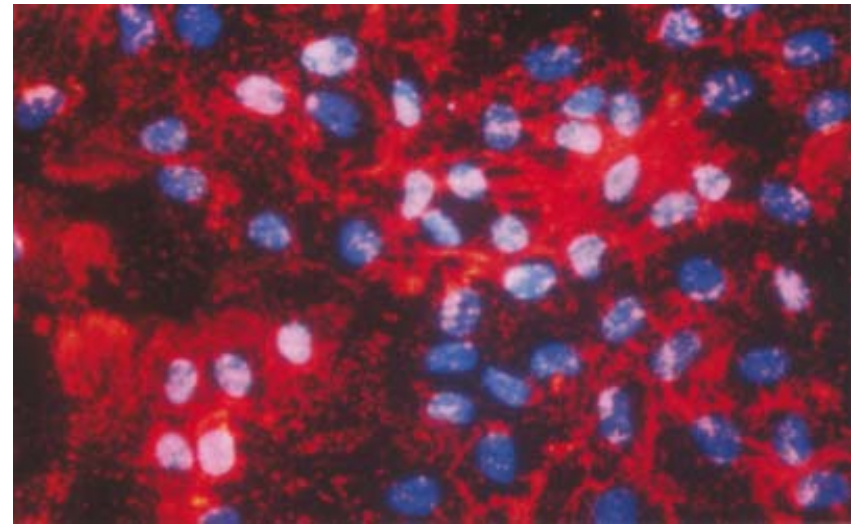

Abb. 5a Durch Optimierung der Isolations- und Kulturbedingungen lassen sich Knorpelzellen in Monolayer-Primärkulturen in ausreichender Zahl und gutem Funktionszustand anzüchten. ImmunfluoreszenzFärbung gegen Chondroitinsulfat.

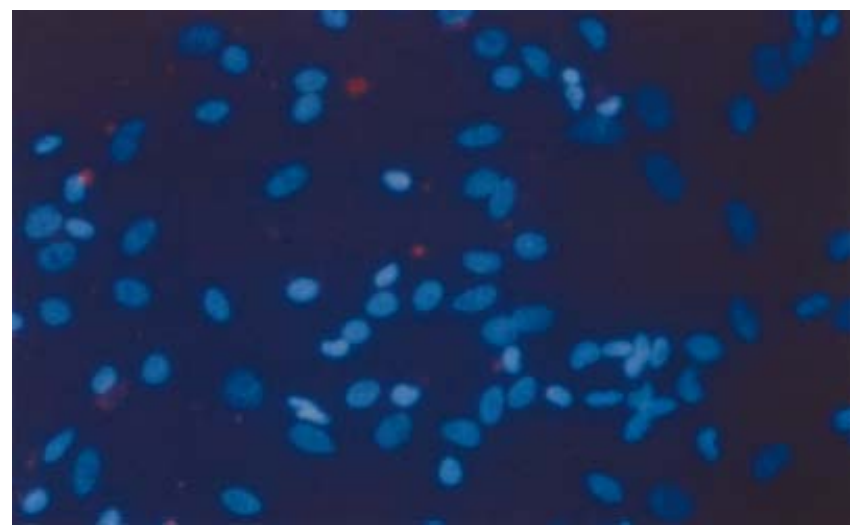

Abb. 5b Hautfibroblasten des gleichen Spenders, kultiviert unter den gleichen Bedingungen, zeigen keine Anfärbbarkeit gegen knorpeltypisches Chondroitinsulfat.

grundsubstanz (Matrix), freigesetzt werden. Das Auslösen von Zellen aus ihrem Ursprungsgewebe mit unterschiedlichen zellbiologischen Techniken wird häufig auch als Zellisolation bezeichnet. Wichtig für die Zellisolation ist, dass sie möglichst schonend für die Zellen durchgeführt wird. Aggressive lsolationsmethoden, insbesondere durch manche Verdauungsenzyme verursacht, können zur Zellschädigung und infolge dessen zu einem unerwünschten Wandel des funktionellen Zustands der Zellen führen. Die Zellisolation muss daher so schonend wie möglich erfolgen.

Fraktionierte Isolationstechniken haben sich in diesem Zusammenhang als vorteilhaft erwiesen (Abb.3a). Vor der eigentlichen Zellanzucht muss in einem weiteren Arbeitsschritt die Nährlösung für die Chondrozytenkultivierung vorbereitet werden.

Hierzu sollte ein für Chondrozyten optimiertes Basismedium verwendet werden. Nicht alle Rezepturen kommerziell erhältlicher Medien sind für die Anzüchtung von Chondrozyten geeignet, da das Wachstum und der funktionelle Zustand der Zellen neben anderen Faktoren mit von den Inhaltsstoffen des verwendeten
Basismediums abhängig ist. Durch bestimmte Kalziumkonzentrationen mancher Medien kann z.B. ein vorzeitiger Verlust chondrozytenrelevanter Stoffwechselleistungen auftreten [11].

Das Basismedium wird zusätzlich mit patienteneigenem Serum ergänzt. Seren tierischer Herkunft sollten aus verschiedenen Gründen nicht verwendet werden. Zum einen könnten hierdurch Infektionen tierischer Herkunft auf den Patienten übertragen werden. Zum anderen hat sich die Verwendung von patienteneigenem Serum im Vergleich zu tierischen Seren als vorteilhaft für das Zellwachstum und hieraus resultierend als günstig für den knorpelspezifischen Funktionsstoffwechsel der Zellen erwiesen. Auch antibiotische Zusätze, wie sie häufig in Zellkulturversuchen Anwendung finden, sollten für die Chondrozytenanzüchtung zu Transplantationszwecken nicht verwendet werden. Diese Substanzen können den Stoffwechsel der Knorpelzellen negativ beeinflussen und sie können die Selektion antibiotikaresistenter Keime fördern. Ebenso kann hierdurch eine mikrobielle Kontamination der Zellkulturen maskiert werden, die in der abschließenden Keimkontrolle vor der Retransplantation nicht unbedingt erfasst wird. 
Im Anschluss zur Zellisolation werden die herausgelösten Chondrozyten dann im vorbereiteten serumkompletierten Vollmedium aufgenommen und in Zellkulturflaschen ausgesät. Die Zellkulturflaschen werden in einen Brutschrank gestellt, der günstige atmossphärische Bedingungen und Temperaturverhältnisse gewährleistet.

Nach einer kurzen Adaptionsphase siedeln sich die Zellen am Boden der Flaschen $\mathrm{ab}$ und beginnen zu proliferieren. Unter Proliferation versteht man die Zellvermehrung durch Zellteilung.

In Abhängigkeit der Zellvermehrung und des hiermit verbundenen Verbrauchs der Nährstoffe muss das verbrauchte Zellkulturmedium regelmäßig gegen frisches Medium ersetzt werden.

Ist eine ausreichende ZellzahI erreicht, sollten kurz vor der Zellernte neben unabdingbaren Sterilitätskontrollen zellund molekularbiologische Qualitätsuntersuchungen durchgeführt werden.

Aufgrund der bis heute vorliegenden experimentellen und klinischen Erfahrungen empfehlen wir die Analyse folgender Qualitätsmerkmale:

1. Lebensfähigkeit, d.h. Zellvitalität

2. absolute Anzahl der zu transplantierenden Zellen

3. zellulärer Differenzierungsgrad und Zellmorphologie

4. zelluläre Sekretionsleistung der wichtigsten Strukturproteine des Knorpels

Zellkulturen, die diese Qualitätskriterien nicht erfüllen, sollten auch nicht transplantiert werden.

\section{Dritter Schritt:}

Die eigentliche Operation erfolgt in Rückenlage des Patienten unter Verwendung einer Blutsperre und bei perioperativer Antibiotika-Prophylaxe. Die Schnittführung wird in Abhängigkeit von der Lokalisation des Knorpeldefektes gewählt und liegt am Kniegelenk medial oder lateral parapatellar. Nach Darstellung des Knorpelschadens erfolgt das sorgfältige Anfrischen des Defektgrundes mittels Skalpell und scharfem Löffel, ohne dass die subchondrale Knochenplatte durchbrochen wird (Abb.6). Somit kann das Einsprießen von Bindegewebszellen und die Ausfüllung des Defekts durch Faserknorpel vermieden werden. Die Blutstillung erfolgt durch Unterspritzen einer

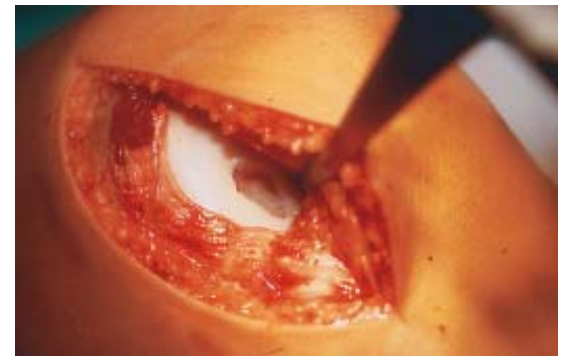

Abb. 6 Darstellen der Defektkammer mit sorgfältiger Anfrischung des Defektgrundes.

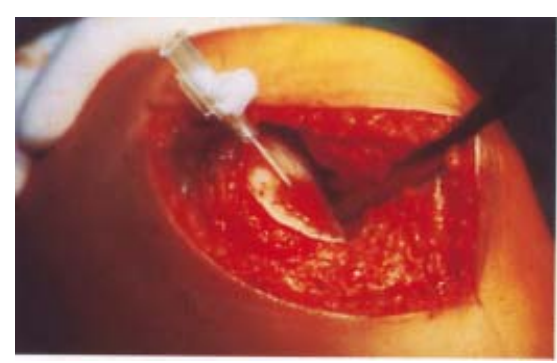

Abb. 7 Kurz vor Zellretransplantation vorsichtig eingelegte Verweilkanüle am oberen Pol des Periostlappens. Es muss absolute Dichtigkeit der Defektkammer angestrebt werden.

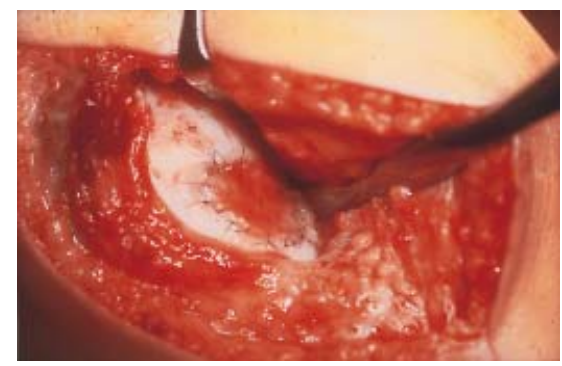

Abb. 8 Mit letzter Naht und Fibrinkleber abgedichteter Periostlappen nach erfolgter Zelltransplantation.

speziellen Substanz, anschließend werden die Knorpelränder bis zu den intakten angrenzenden Arealen geglättet, wobei eine ausreichende „Knorpelschulter“ erhalten werden sollte. Jetzt wird der Periostlappen vom körpereigenen Schienbein innenseitig kniegelenksnah entnommen, wobei eine Sterilfolie als Muster für die Ausdehnung des Knorpeldefektes verwendet werden kann.

Die Lappengröße wird so gewählt, dass sie den Defekt nach allen Seiten um ca. $2 \mathrm{~mm}$ überragt.

Danach präpariert man den Lappen durch Umschneidung und vorsichtiges Abheben mit einem Raspatorium. Der Periostlappen wird mit einem PDS-Faden 6,0 in Form von Einzelknopfnähten fixiert, wobei bis auf die eingelegte Ver- weilkanüle Größe 14 am oberen Pol absolute Dichtigkeit angestrebt wird (Abb. 7) Notwendig sind während dieser Prozedur das Befeuchten des Periostlappens, das Abdichten der Naht mittels Fibrinkleber und die Probeinjektion von Kochsalz zur Dichtigkeitsprüfung. Nach Absaugen der Kontrollflüssigkeit wird die Knorpelzellsuspension mit einer „Insulinspritze“ aus dem sterilen Transportgefäß aspiriert und über die Verweilkanüle unter den Lappen injiziert. Am Ende erfolgt das Entfernen der Verweilkanüle, der Verschluss des Restdefektes mit letzter Naht und Fibrinkleber (Abb.8), der Wundverschluss über Redondrainagen und die postoperative Lagerung auf einer Motorschiene.

Operatives Vorgehen bei der ACT:

- Darstellen des Knorpeldefekts

- Präparation der Defektkammer

- sorgfältige Blutstillung

- Entnahme und Präparation des Periostlappens

- Deckelung der Defektkammer mit dem Periostlappen

- sorgfältige Dichtigkeitsprüfung des Periostlappens

- Injektion der Knorpelzellen in die Defektkammer

- letzte Naht und wasserdichte Fibrinversiegelung des Periostlappens

\section{Begleit- und Nachbehandlung}

Bezüglich des Bewegungsumfanges des Kniegelenkes wird keine Einschränkung vorgegeben, 6 Wochen darf allerdings nur mit Bodenkontakt belastet werden, danach ist für weitere 6 Wochen eine zunehmende Belastungssteigerung bis zur Vollbelastung erlaubt. Anschließend ist die Wiederaufnahme von Sportarten ohne starke Kniegelenksbelastung, wie z.B. das Radfahren und Schwimmen möglich, Sprungsportarten sollten für die Dauer eines halben Jahres gemieden werden.

\section{Eigene Erfahrungen mit der ACT}

Zwischen Juli 1998 und Februar 2000 wurden in der BG-Unfallklinik Tübingen bei insgesamt 28 Patienten eine ACT am Kniegelenk durchgeführt. Es handelte sich bei den Patienten um 19 Männer und 9 Frauen, die jüngste Patientin war 18 Jahre alt, der älteste Patient 51, das Durchschnittsalter betrug 29,8 Jahre.

Bei 16 Patienten war der umschriebene Knorpeldefekt traumatischer Genese, bei 10 Patienten lag eine Osteochondrosis dissecans und bei 2 Patienten lagen um- 
schriebene Defekte degenerativer Ursache vor. 2 der 28 Patienten waren in der Vergangenheit in einer anderen Klinik erfolglos mit einer Pridiebohrung versorgt worden. In 12 Fällen lag ein Defekt der medialen Femurkondyle, in 8 Fällen einer an der lateralen Femurkondyle, in 4 Fällen ein Defekt retropatellar und in 4 weiteren Fällen einer im femoralen Gleitlager vor. Die Defektgröße schwankte zwischen 2 und $11 \mathrm{~cm}^{2}$. Die Defekttiefe betrug in keinem Fall mehr als $7 \mathrm{~mm}$. Voraussetzungen für die Transplantation waren intakte Band- und Meniskusverhältnisse, ebenfalls durften keine wesentlichen Abnutzungserscheinungen (beginnende Gonarthrose) vorliegen. Die Knorpelentnahme wurde bei allen Patienten arthroskopisch vorgenommen. Bei dieser Arthroskopie wurde die Indikation zur Knorpelzelltransplantation nach den oben genannten Kriterien nochmals überprüft. Bei 13 weiteren zur ACT indizierten Patienten mußte aufgrund des intraoperativen Befundes auf eine autologe Knorpelzelltransplantation verzichtet werden. Bei 7 dieser Patienten wurde zur Behandlung der Knorpeldefekte die weiter oben beschriebene Mosaikplastik durchgeführt. Die Knorpelentnahme zur Zellanzüchtung wurde anfangs mit einer Kürette aus dem parapatellaren Gleitlager entnommen, viel schonender und einfacher zeigte sich jedoch die Entnahme aus der Notchregion mit einem kleinen Stanzzylinder.

Die Anzüchtungszeit der Chondrozyten konnte durch Optimierung der Isolations- und Zellkulturbedingungen von inital 3 Wochen auf durchschnittlich 11 Tage reduziert werden. Die begleitend durchgeführten mikrobiologischen Untersuchungen ergaben bei keinem Patienten einen Keimnachweis in den Kulturen. Nach Erreichen der erforderlichen ZeIIzahI wurden zellbiologische Qualitätsuntersuchungen durchgeführt und der Patient zur Implantation der Zellen kurzfristig wieder einbestellt. Postoperativ wurde eine Redondrainage ohne Sog intraartikulär für 2 Tage belassen.

Die Nachbehandlung erfolgte wie oben beschrieben. Postoperative Komplikationen traten bei 2 Patienten auf.

Bei einem Patienten verblieb nach 6 Wochen ein erhebliches Beugedefizit, so dass eine Rearthroskopie zur Arthrolyse notwendig wurde. Hierbei zeigte sich der Periostlappen vollständig eingeheilt, zu sehen war eine völlig glatte, allerdings noch weiche Oberfläche, Nähte waren nicht mehr sichtbar. Nach der Arthrolyse war der weitere Verlauf komplikationslos. Bei einer weiteren Patientin trat 5 Tage nach der Implantation eine schmerzhafte Kniegelenksschwellung mit leicht erhöhten Entzündungswerten auf. Die durchgeführte Rearthroskopie zeigte einen weitgehend abgelösten Periostlappen der arthroskopisch entfernt werden mußte. Eine Infektion konnte vermieden werden, der weitere Verlauf war komplikationslos. Bei den übrigen 26 Patienten konnten keine Besonderheiten während des postoperativen Verlaufs beobachtet werden.

Die weiteren Verlaufskontrollen erfolgen derzeit in regelmäßigen Abständen nach dem von der Cartilage Repair Society empfohlenen Dokumentationsbogen. In der subjektiven Einschätzung wurde von den 28 transplantierten Patienten von 17 bis dato vollständige Beschwerdefreiheit angegeben, 7 klagen über Restbeschwerden bei deutlicher Beschwerdereduktion, 3 Patienten empfinden Beschwerdepersistenz und eine Patientin klagt über eine Zunahme der Beschwerdesympthomatik. Die 2 in Vergangenheit mit einer Pridiebohrung vorbehandelten Patienten waren nach ACT zum ersten Mal nach Trauma beschwerdefrei. Einer der beiden transplantierten Patienten mit lokal begrenzten Knorpeldefekten degenerativer Ursache gehört zur Gruppe der Patienten mit Beschwerdepersistenz, die Patientin mit oben beschriebener Revision klagt über eine Zunahme der Beschwerden. Von 5 innerhalb des ersten Jahres nach ACT arthroskopisch nachuntersuchten Patienten wiesen drei eine vollständig glatte Oberfläche auf (Abb.9). Die 3 Patienten mit arthroskopisch gutem Ergebnis gehören in die Gruppen beschwerdefrei oder wenig Beschwerden. Die beiden Patienten mit rauher Regeneratoberfläche gehören zur Gruppe der Patienten mit Beschwerdepersistenz. Nur diese 5 der insgesamt 28 transplantierten Patienten waren allerdings mit einer arthroskopischen Verlaufskontrolle bisher einverstanden.

Vorläufige Auswertung der eigenen Ergebnisse nach ACT

- unter strenger Indikationsstellung gute Ergebnisse bei geringer Komplikationsrate

schlechte Ergebnisse bei auch nur lokal begrenzten Defekten degenerativer Ursache

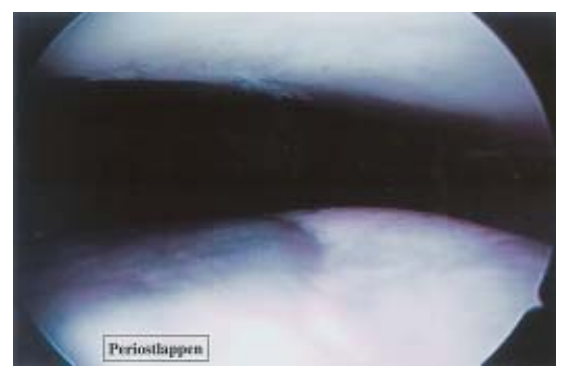

Abb.9 Gute Defektauffüllung mit glatter Oberfläche 3 Monate nach autologer Knorpelzelltransplantation.

\section{Diskussion}

Die Behandlung von Knorpelschäden bekannter und unbekannter Ursache in verschiedenen Gelenken stellt nach wie vor ein großes Problem dar. Im klinischen Alltag wird derzeit eine ganze Reihe unterschiedlichster Verfahren zur operativen Behandlung von Knorpeldefekten eingesetzt. Der Einsatz des gewählten Verfahrens sollte hierbei vor allem durch das Beschwerdebild, das Defektausmaß, die Defektlokalisation und durch die Defektursache begründet sein.

Bei kleineren begrenzten Knorpelschäden kann oft durch die arthroskopische Gelenkspülung mit gleichzeitiger Entfernung von irritierenden Knorpelabschilferungen Beschwerdefreiheit erreicht werden. Bei überlastungsbedingten Knorpelschäden infolge Achsfehlstellungen haben sich gelenknahe Osteotomien zur Korrektur der Fehlstellung bewährt [10]. Die operative Druckentlastung des betroffenen Gelenkkompartiments führt häufig zur Schmerzreduktion und einer Verbesserung der Gelenkfunktion. Röntgenologisch kann in manchen Fällen sogar ein Wiederaufbau des Gelenkspalts beobachtet werden.

Bestehen höhergradige Knorpelläsionen können wiederum, unter Berücksichtigung von Defektausmaß und -ursache, unterschiedliche Verfahren angewandt werden.

Die Wiederbefestigung eines größeren, abgelösten Knorpel- oder Knorpelknochenfragments im Defektlager wird seit längerem mit den unterschiedlichsten Methoden und Ergebnissen durchgeführt. Generell hat die Refixation eines Knorpelknochenfragments bessere Heilungsaussichten als die eines Knorpelfragments ohne Knochenanteil. 
Die bereits beschriebene Pridiebohrung oder das „mikrofracturing“ führt in der Regel im Bereich der Defektzone zur Ausbildung eines minderwertigen Narbengewebes.

Insgesamt gilt, dass die beiden kortikalispenetrierenden Verfahren bei traumatisch induzierten Knorpeldefekten bessere Ergebnisse erzielen als bei Defekten aufgrund einer Osteochondrosis dissecans oder degenerativen Veränderungen.

Die arthroskopisch durchführbare Transplantation einzelner oder mehrerer autologer Knorpel-Knochenzylinder erzielt unter Berücksichtigung der bis heute vorliegenden Fallzahlen bei korrekter operativer Technik gute klinische Ergebnisse [5].

Aufgrund der begrenzten Verfügbarkeit autologen Spendermaterials und der abnehmenden Transplantatstabilität im großen Defektlager sind jedoch auch dieser Methode Grenzen gesetzt.

Mit der alleinigen Periost- oder Perichondriumtransplantation können zwar größere Defektareale behandelt werden, aber auch hier entsteht, wie bei den oben beschriebenen Verfahren, kein originärer hyaliner Knorpel, sondern allenfalls ein Mischknorpel im ehemaligen Defektbett.

In einer erst kürzlich veröffentlichten Studie berichten Peterson u. Mitarb. über die klinischen, arthroskopischen und histologischen Resultate von 94 Patienten, die innerhalb von 2 bis 9 Jahren nach autologer Knorpelzelltransplantation nachuntersucht werden konnten [13]. Gute bis sehr gute klinische Ergebnisse wurden von den Autoren bei folgenden Defektlokalisationen beschrieben: 94\% bei isolierten Defekten der Femurkondylen, $67 \%$ bei multiplen Läsionen, 89\% bei Osteochondrosis dissecans, 65\% bei Knorpeldefekten der Patella, und 75\% bei Defekten der Femurkondyle mit gleichzeitigem Ersatz des vorderen Kreuzbandes. Bei 53 arthroskopisch nachuntersuchten Patienten zeigte sich eine gute Defektauffüllung mit Adhärenz zur darunterliegenden subchondralen Knochenlamelle und saumloser Integration zum umgebenden Knorpel.

Die histologischen Ergebnisse von 37 durchgeführten Biopsien zeigten die Korrelation zwischen guten klinischen Ergebnissen und der Ausbildung eines hyalinartigen Knorpels im ehemaligen Defektbett.
Die insgesamt doch sehr guten Ergebnisse mit Erfolgsquoten bis über 90\% konnten in der Qualität, wie sie von Brittberg und Peterson berichtet werden, von anderen nationalen und internationalen Anwendern der ACT bisher nicht immer bestätigt werden.

Die Problematik in der Beurteilung der unterschiedlich berichteten klinischen und histologischen Ergebnisse nach ACT ist allerdings vielschichtig. Die Ergebnisdokumentation ist in den bisher vorliegenden Studien wenig einheitlich.

In der klinischen Auswertung wurden zum Teil die unterschiedlichsten Scores verwendet, die häufig nur schwer miteinander vergleichbar sind. In der Cartilage Repair Society hat man sich daher vor kurzem auf einen, wenn auch sehr komplizierten, Dokumentationsbogen einigen können, der eine Vereinheitlichung der Nachuntersuchungen herbeiführen soll.

In manchen der zur ACT veröffentlichten Studien ist es schwierig zu beurteilen, ob es sich bei den vorgestellten histologischen Präparaten um repräsentative Schnitte handelt, oder ob nur Schnitte im Sinne einer statistisch nicht korrekten Positiv- oder Negativauswahl gezeigt werden.

In den wenigsten Veröffentlichungen wurden ausreichende Angaben darüber gemacht, was für ein Kultivierungsprotokoll für die Zellanzüchtung verwendet wurde und ob irgendwelche zell- oder molekularbiologischen Untersuchungen zur Qualitätssicherung des Knorpelzelltransplantats vor Retransplantation durchgeführt wurden. Ferner fehlen neben Angaben zur Zellvitalität und dem funktionellen Zustand der transplantierten Zellen meistens die Daten zur absoluten Anzahl transplantierter Knorpelzellen pro Defektvolumen.

In einer 1997 veröffentlichten In-vitroStudie konnte nachgewiesen werden, dass auch bei der ACT eine Dosis-Wirkungs-Beziehung der Zelldichte bezüglich einer optimalen Matrixneuformation existiert [14].

Das Fehlen der Angaben zu wichtigen zell- und molekularbiologischen Eigenschaften läßt keinerlei Einschätzung der Qualität eines verwendeten Zelltransplantats zu. Dadurch ist es auch nicht möglich zu beurteilen, inwieweit ein schlechtes Transplantationsergebnis nach ACT auf eine unzulängliche Transplantatqualität zurückzuführen ist, oder ob es sich um ein Versagen der ACT als Methode handelt.

Neben der Zellqualität und der korrekten operativen Technik ist aus den teilweise bereits genannten Gründen die richtige Indikationsstellung von entscheidender Bedeutung für das Ergebnis der ACT $[10,15,16]$. In einer erst kürzlich veröffentlichten Studie konnte nachgewiesen werden, dass 23 von 24 Patienten, bei denen die Indikation zur ACT gestellt wurde, nicht für dieses Verfahren geeignet waren. Bei 15 (63\%) dieser 24 Patienten bestanden sogar multiple Kontraindikationen [17].

Dass in solchen Fällen falscher Indikationsstellung schlechte klinische und histologische Ergebnisse nach ACT resultieren, ist nicht weiter verwunderlich.

Aufgrund der geschilderten Problematik wird daher von unterschiedlichen Autoren die Etablierung von allgemeingültigen Qualitätsstandards gefordert $[15,18]$, um zum einen die Transplantationsergebnisse der einzelnen Studien besser miteinander vergleichen zu können und um zum anderen der optimierten Herstellung der Zellpräparate gerecht zu werden.

Zusammenfassend läßt sich aus unserer Erfahrung heraus festhalten, dass die autologe Knorpelzelltransplantation bei korrekter lndikationsstellung, adäquater Transplantatqualität und Operationstechnik ein vielversprechendes und ergänzendes Verfahren in der Behandlung von artikulären Knorpeldefekten darstellt. An der Berufsgenossenschaftlichen Unfallklinik Tübingen wird die ACT daher vor allem bei jungen Patienten mit großen Knorpeldefekten traumatischer Ursache eingesetzt, bei denen die weiter oben beschriebenen Verfahren, wie z. B. die Mosaikplastik, erfahrungsgemäß überfordert sind.

Da das Ergebnis der autologen Knorpelzelltransplantation jedoch von einer Vielzahl unterschiedlicher und komplexer Faktoren abhängig ist, kann ihre Anwendung nur von hierfür ausgebildeten Ärzten und an spezialisierten Zentren unter Gewährleistung der beschriebenen Qualitätskriterien empfohlen werden. 


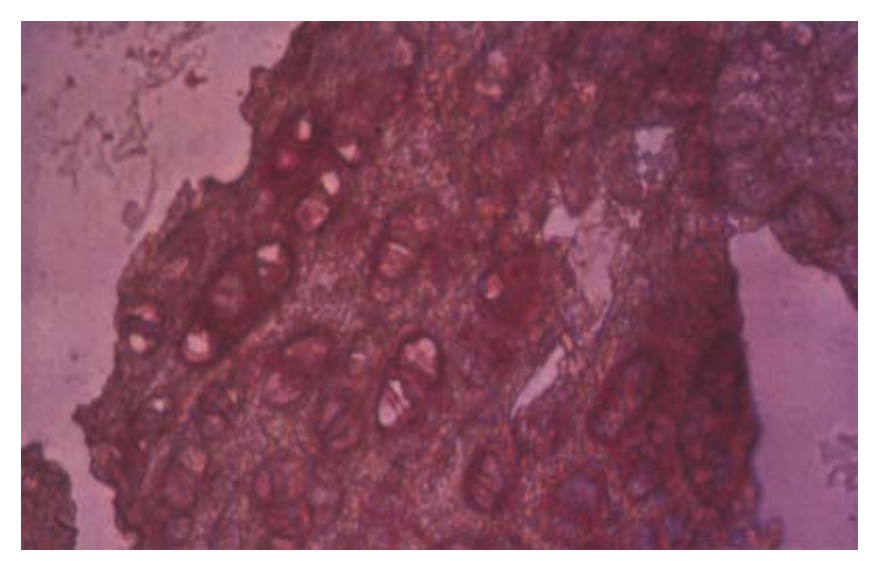

Abb.10 KollagenTyp-Il-Vlies mit humanen Gelenkknorpelzellen besiedelt. Die Zellen ordnen sich im Vlies in Form von Chondronen an, wie man sie vom nativen Gelenkknorpel her kennt. Peroxidasefärbung mit spezifischem Antikörper gegen Proteoglykan.

\section{Ausblick}

Bei vollständiger Degeneration des gelenküberziehenden Knorpels im Sinne der manifesten Osteoarthrose sind alle bisher zur Verfügung stehenden Verfahren zur biologischen Rekonstruktion als weitgehend erfolglos zu bezeichnen. In diesen Fällen verbleibt zur Erhaltung der Gelenkfunktion letztendlich nur der alloplastische Gelenkersatz. Das Ziel der wissenschaftlichen Bemühungen im Bereich der Herstellung eines bioartifiziellen Oberflächenersatzes ist es daher, neben der Verbesserung der klinischen und histologischen Ergebnisse für die ACT mit ihrem begrenzten Anwendungsspektrum, eine Erweiterung der Indikationsstellung für biologische Rekonstruktionsverfahren von Oberflächendefekten in unterschiedlichen Gelenken zu erreichen.

Die verschiedenen, häufig interdisziplinären Teilschritte zur Herstellung eines biologischen Implantats für die rekonstruktive Chirurgie subsumiert man in neuerer Zeit unter dem Begriff „Tissue Engineering“. Aus wissenschaftlicher Sicht versteht man hierunter die künstliche Herstellung bioartifizieller Konstrukte oder Gewebe aus lebenden Zellen bzw. Zellmatrix und Biomaterialien.

Grundvoraussetzung für die erfolgreiche klinische Anwendung des biologischen Oberflächenersatzes ist die Herstellung einer funktionsfähigen Knorpelmatrix, die in der Lage ist, mit dem subchondralen Knochen $\mathrm{zu}$ fusionieren und deren biomechanische Eigenschaften auch langfristig mit denen des hyalinen $\mathrm{Ge}$ lenkknorpels vergleichbar sind.

Nach den Ergebnissen der gegenwärtig vorliegenden In-vitro- und In-vivo-Studien ist hierzu eine ausreichende Zahl vitaler knorpelbildender Zellen in phänotypisch stabilem Funktionszustand und in einer mechanisch stabilen Primärmatrix erforderlich [19].

Die primäre Matrix, also das Trägermaterial, dient den Zellen als temporäre Leitstruktur für ihre räumliche Ausrichtung und bis zur Resorption des Trägers als Ort der eigenen Matrixsynthese. Da der Phänotyp, d.h. die funktionellen Eigenschaften, vor allem durch Zell-Matrix-Interaktionen beeinflusst wird, ist die $\mathrm{Zu}$ sammensetzung des Trägermaterials mit von entscheidender Bedeutung für die spätere Regeneratqualität in vivo.

Das verwendete Trägermaterial stellt somit einen essentiellen Parameter für die Herstellung eines funktionellen Knorpeltransplantats dar. Weiterhin sind seine biochemischen und physikalischen Eigenschaften entscheidend für die operative Handhabbarkeit und die Transplantatintegration in das Wirtsgewebe. Zur Herstellung eines optimierten Knorpelimplantats werden derzeit verschiedene Trägermaterialien in vitro und in vivo getestet, wobei im Wesentlichen entweder natürlich vorkommende Makromoleküle und/oder abbaubare synthetische Polymere Verwendung finden (Abb.10).

Neben der Beschaffenheit des Trägermaterials wird das Proliferations- und Differenzierungsverhalten von Knorpelzellen auch von einer Reihe verschiedener Wachstumsfaktoren beeinflusst. Ausgehend von der embryonalen Entstehung des Skelettsystems, bis hin zu Reparaturprozessen im erwachsenen Organismus sind diese Botenstoffe in alle Teilaspekte des Wachstums und Stoffwechsels involviert $[20,21]$. In experimentellen Ansätzen zur bioartifiziellen Herstellung von Knorpel und Knochen wird daher durch den Einsatz unterschiedlicher Wachs- tumsfaktoren versucht, den Stoffwechsel potentiell knorpel- oder knochenbildender Zellen zu optimieren.

Während Faktoren mit hoher zellvermehrungsinduzierender Wirkung, wie z.B. der basische Fibroblastenwachstumsfaktor (bFGF), vor allem zur Gewinnung von Zellmasse und zur Differenzierungsvorbereitung eingesetzt werden $[19,22]$, finden Faktoren der sogenannten Superfamilie der transformierenden Wachstumsfaktoren beta (TGF- $\beta$ ) und hier insbesondere die morphogen wirkenden BMPs (bone morphogenetic protein) Anwendung in der Differenzierungsinduktion und der Stimulation der spezifischen Synthese von Knorpelgrundsubstanz [19, 21,23].

Weitere wichtige Regulatoren des Knorpelstoffwechsels sind nach den bisherigen wissenschaftlichen Erkenntnissen der Sauerstoffpartialdruck, mechanische Einflüsse und bestimmte Elektrolytkonzentrationen im Umfeld der Knorpelzellen $[11,19,24]$.

Ob unter Verwendung von angezüchteten Knorpelzellen oder differenzierungsfähigen Stammzellen durch eine Optimierung der aufgezählten Regulatoren ein effizientes biohybrides Knorpelimplantat für die erweiterte therapeutische Anwendung hergestellt werden kann, wird momentan von unterschiedlichen Arbeitsgruppen weltweit erforscht. Darüber hinaus wird in verschiedenen experimentellen Ansätzen versucht, ein kombiniertes Knorpel-Knochenimplantat zu entwickeln, da sich die Fusion eines reinen und vor allem reifen Knorpelimplantats mit dem subchondralen Knochen, ähnlich wie bei der Refixation eines reinen Knorpelfragments ohne knöchernen Anteil, als problematisch erwiesen hat [19]. Sollten die wissenschaftlichen Bemühungen erfolgreich sein, hätte dies eine neue Ära in der Behandlung fortgeschrittener artikulärer Knorpeldefekte zur Konsequenz.

\section{Literatur}

${ }^{1}$ Brittberg M, Lindahl A, Nilsson A, Ohlson C, Isaksson $\mathrm{O}$, Peterson L. Treatment of deep cartilage defects in the knee with autologous chondrocyte transplantation. N Engl Med 1994: 331: 889-895

2 Richardson JB, Caterson B, Evans EH, Ashton BA, Roberts S. Repair of human articular cartilage after implantation of autologous chondrocytes. J Bone Joint Surg Br 1999; 81: $1064-1068$

${ }^{3}$ Steinwachs MR, Errgelet C, Lahm A, GuhlkeSteinwachs U. Klinische und zellbiologische 
Aspekte der autologen Chondrozytenimplantation. Unfallchirurg 1999; 102: 855 860

${ }^{4}$ Minas T, Nehrer S. Current concepts in the treatment of articular cartilage defects. Orthopedics 1997; 20: 525-538

${ }^{5}$ Hangody L, Kish G, Karpati Z, Udvarhelyi I, Szigeti I, Bely M. Mosaicplasty for the treatment of articular cartilage defects: application in clinical practice. Orthopedics 1998; 21: $751-756$

${ }^{6}$ Bayreuther K, Rodemann HP, Hommel R, Dittmann K, Albiez M, Francz PI. Human skin fibroblasts in vitro differentiate along a terminal cell lineage. Proc Natl Acad Sci 1988; 85: 5112-5116

${ }^{7}$ Sell S, Gaissmaier C, Herr G, Esenwein S, Küsswetter W, Fritz J, Wittkowski KM, Rodemann HP. Different Behavior of Human Osteoblast-like Cells Isolated from Normal and Heterotopic Bone In Vitro. Calcif Tissue Int 1998; 62: $51-59$

${ }^{8}$ Benya PD, Shaffer JD. Dedifferentiated Chondrocytes reexpress the differentiated Collagen Phenotype when cultured in Agarose Gels. Cell 1982; 30: 215-224

${ }^{9}$ Gaissmaier C, Fritz J, Maurer F, Aicher W, Höntzsch D, Weise K. Inkomplette Redifferenzierung humaner artikulärer Knorpelzellen in Alginatbeads nach mehrfacher Passagierung in der Monolayerkultur. Fortschrittsberichte der 64. Jahrestagung der DGU, 10.-13. September 2000, Hannover

${ }^{10}$ Gaissmaier C, Fritz J, Müller JE, Krackhardt T, Rether JR, Höntzsch D, Weise K. Autologe Knorpelzelltransplantation - Indikation und Technik. Akt Traumatol 1998; 28 : $245-250$

${ }^{11}$ Flechtenmacher J, Koyano Y, Hejna M, Schmid TM, Puhl W, Mollenhauer J. Do calcium and zinc ions influence matrix mole- cule synthesis of chondrocytes. Z Orthop 1996; 134: 283-289

${ }^{12}$ Weisl H. Intertrochanteric osteotomy for osteoarthritis. A long term follow-up. J Bone Joint Surg 1980; 72-B: 37-42

${ }^{13}$ Peterson L, Minas T, Brittberg M, Nusson A, Sjogren-Janssson E, Lindahl A. Two- to 9year outcome after autologous chondrocyte transplantation of the knee. Clin Orthop 2000; 374: $212-234$

${ }^{14}$ Chen AC, Nagrampa JP, Schinagl RM, Lottmann LM, Sah RL. Chondrocyte transplantation to articular cartilage explants in vitro. J Orthop Res 1997; 15: 791 - 802

${ }^{15}$ Schneider U, Breusch SJ, von der Mark K. Aktueller Stellenwert der autologen Chondrozytentransplantation. Z Orthop 1999; $386-392$

${ }^{16}$ Nehrer S, Spector M, Minas T. Histologic analysis of tissue after failed cartilage repair procedures. Clin Orthop 1999; 365: 149162

${ }^{17}$ Mont MA, Jones LC, Vogelstein BN, Hungerford DS. Evidence of inappropriate application of autologous cartilage transplantation therapy in an uncontrolled environment. Am J Sports Med 1999; 27: 617-620

${ }^{18}$ Weise K, Fritz J, Hörnlein RS, Krackhardt T, Gaissmaier C. Die autologe Chondrocytentransplantation. Grundlagen, aktueller Stand und Ausblick. Deutsche Gesellschaft für Unfallchirurgie (DGU), Mitteilungen und Nachrichten 1999; 39: 28-38

${ }^{19}$ Freed LE, Martin I, Vunjak-Novakovic G. Frontiers in tissue engineering. Clin Orthop 1999; 367; 46-58

${ }^{20}$ Reddi AH. Bone and cartilage differentiation. Curr Opin Genet Dev 1994; 4: 737-744

${ }^{21}$ Erlacher L, Ng CK, Ullrich R, Krieger S, Luyten FP. Presence of cartilage-derived morphogenetic proteins in articular cartilage and en- hancement of matrix replacement in vitro. Arthritis Rheum 1998; 41: 263-273

${ }^{22}$ Murakami S, Kan M, McKeehan WL, de Crombrugghe B. Up-regulation of the chondrogenic Sox9 gene by fibroblast growth factors is mediated by the mitogen-activated protein kinase pathway. Proc Natl Acad Sci 2000; 243: 187-191

${ }^{23}$ Flechtenmacher J, Huch K, Thonar E, Mollenhauer JA, Davies SR, Schmid TM, PuhI W, Sampath TK, Aydelotte MB, Kuettner KE. Recombinant human osteogenic protein 1 is a potent stimulator of the synthesis of cartilage proteoglycans and collagens by human articular chondrocytes. Arthritis Rheum 1996; 39: 1896-1904

${ }^{24}$ Krackhardt T, Gaissmaier C, de Zwart PM, Fritz J, Höntzsch D, Weise K. Cytosolic free calcium inhibits the chondrocyte-derived growth factor matrix synthesis in chondrocytes. Fortschrittsberichte des 4th European Trauma Congress, 10. - 13. September 2000, Hannover

Prof. Dr. med. K. Weise

Ärztl. Direktor

T. Krackhardt

Oberarzt

Ch. Gaissmaier

Assistenzarzt

BG-Unfallklinik Tübingen

Schnarrenbergweg 95

72076 Tübingen 